\title{
Older Adults Research in Acute Care - Breaking the Barriers
}

\author{
Angela P. Halpin ${ }^{1}$, Felicia S. Hodge ${ }^{1,2}$, and Puiyen Chui ${ }^{1}$ \\ University of California, Los Angeles, School of Nursing \\ University of California, Los Angeles, School of Public Health
}

\section{Introduction}

Population-based growth has familiarized health care providers with the special needs of older patients. We know the elderly, those 65 years or older, represent $13.7 \%$ of the U.S. population, with a projected rise to $19 \%$ by 2030 (Administration on Aging, 2013). Yet, the Cumulative Index to Nursing and Allied Health indicates that only $3 \%-6 \%$ of clinical trials are specific to the older adult aged population (Orwig, Williams, \& Martin, 2011). Older adult patients in acute care hospitals (ACH) are known to have multiple comorbidities that include respiratory ailments, cardiac diseases, falls, cognitive impairments, and depression (Formiga et al., 2012; CDC, 2014). Studies have demonstrated that comorbid conditions increase with age (Formiga et al., 2012; Barnett et al., 2012).

Clearly research specific to older adults is under-represented, especially in acute care settings. To date, a search using the terms "older adults", "RCTs", “transitions", and "decision making in acute care settings" retrieved 31 studies in Melvyl and 281 RCTs in PubMed. Generally speaking, these studies used demographic descriptive data such as age, yet most did not disaggregate the older adults for finer grained analyses. For example, RCTs that focus on the transition of older adults from hospitals to home often ignore evaluating how multiple diagnoses impact patients' recovery (Barnett et al., 2012; Naylor, 2011; Naylor et al., 1999). The advanced age of some patients in randomized controlled trials (RCTs) is rarely acknowledged when examining patients' healthcare decision-making. Therefore, studies are recommended to further understand patients' decision-making processes at enrollments and transitions (Hewner, 2014;
Barnett et al., 2012). We believe studying older adults' decision-making in hospital settings is important for the reasons described above, because their mental capacity in such situations might be impaired.

Pneumonia (PN) is a significant co-morbid condition often seen in older adults. Research focusing on the care choices of older adults with pneumonia is timely due to increased readmission rates following discharge. Respiratory illnesses, pneumonia, asthma, and chronic obstructive pulmonary disease, for example, impact variations in oxygen saturations, and affect mental capacities (Aujesky \& Fine, 2008). When studying older adults, the admitting diagnoses need to be considered during enrollment.

This editorial presents our personal experiences related to the recruitment and enrollment of older adults with pneumonia into a RCT, and the barriers and successes experienced implementing this study within acute care hospitals $(\mathrm{ACH})$. We suggest that health care providers in ACHs consider factors influencing older adults' participation in RCTs to better realize and address barriers, and to further the possibilities of important research with this underserved population. Key issues related to a RCT in an ACH that are discussed here are diversity, informed consent, bias, and patient dialogue.

\section{Diversity}

Older patients are hardly a homogenous group, with language variations and wide ranges of mental, personality, and physiological reference points. Personality is defined as individual variations in characteristic patterns of thought, emotions, and behaviors (American Psychological 
Association (APA), (2014). We found that some participants were less engaged in the study due to their illness, while others were more engaged. For example, one patient who was 99 years of age was astute, able to clearly state his interests, family members, and answers to math questions. His personality and mental clarity influenced his decision to enroll, while others were less interested in participating. Those less interested had multiple reasons, such as feeling too ill or too overwhelmed with the hospitalization experience, or they simply expressed no interest. Older adults over the age of 65 present challenges to the researcher because each has a different social experience, based on the extent of each patient's relationship with family and friends.

Cacioppo and Cacioppo (2014) reflected that many older adults have minimal to no source of social support, which impacts their health outcomes and transition decisions to participate in research, as well as care planning after hospitalization. We found that some participants had good social support while many others had very little to no social support, which affects readmission outcomes. One 71-year-old, unmarried participant had no support and was readmitted two additional times in a 60-day period.

According to McHenry, Insel, Einstein, Vidrine, Koerner, and Morrow (2012), variations in cultural norms among the elderly may influence their responses and receptiveness to research. For example, some participants during enrollment were observed discussing the decision to participate with their family prior to enrolling. In our study, we also found, even with the English fluency requirement, that there was an ethnically diverse sample of European, Middle Eastern, Asian, African American, and Hispanic patients. Some potential participants were identified as English speaking, yet did not have fluency when interviewed. Therefore, it is important to consider variations in participants' cultural norms when analyzing outcomes, because variations are known to occur based on patients' cultural, and/or language backgrounds.

\section{Decision Capacity}

Protection of privacy, confidentiality, and study content depends on institutional review board (IRB) approvals. Research presents ethical dilemmas and questions of justice and beneficence, principles that ensure no burden is unduly imposed on any population, honoring autonomous decisions when the capacity to behave autonomously is demonstrated. Research in ACHs includes use of approved screening tools such as the Mini Mental State Exam (MMSE). The established criteria for eligibility require that the potential subject complete the MMSE to evaluate decision-making capacity prior to informed consent (Folstein \& Folstein, 1975). According to Tate et al. (2014), pneumonia often results in a high incidence of cognitive impairment (CI) in the elderly. In our study, $26.2 \%$ of 766 patients screened were found to have some type of CI and thus did not meet eligibility criteria for enrollment. After screening, RNs were notified of the MMSE scores and findings, so other providers could be informed and thus manage mental status changes.

\section{Patient Dialogue}

An unchanging script allows the researcher to focus on professional delivery when providing information about study participation. The IRB approves the script to ensure consistency and protection of subjects. Decision-making capacity is limited to short periods of time in severe illness. However, decision-making capacity must be present at the time of enrollment. For example, when subjects complete the MMSE, the researcher behaves respectfully in reaction to both successful and unsuccessful exam outcomes. To maintain participant dignity, the researcher acknowledges results without causing alarm or shame. Simple statements are useful: "Thank you for your help-that's all I need," "Your RN will review the test result with your physician," and "During an illness, memory can be affected due to changes in your health." These statements 
should be practical to the study's population. When decision-making capacity is absent, the researcher communicates MMSE findings with nursing or medical staff. Keeping the research team and the nursing or medical staff informed through verbal reports or written updates can facilitate communication.

\section{Bias}

Bias consists of the subjective, personal beliefs of staff and researchers. When bias is untamed, integrity of the study becomes compromised. Integrity, or trustworthiness, of a study is defined as the "sum of its credibility, data interpretations, stability of data over time, its conditions, the potential for agreement between two or more independent people about the accuracy of the data, and its generalizability" (Polit \& Beck, 2011; Waltz, Stickland, \& Lenz, 2010). RCTs are known to use non-blinded and double-blinded studies to prevent variations in outcomes. According to Polit and Beck (2011), blinded designs and protocol steps are attentive to potential bias by protecting outcome variation via random allocation of interventions.

Blinding reduces biasing of perception when the treatment assignment is not known to certain individuals, thus protecting the study integrity and credibility (Fain, 2013). Bias in our study was controlled by the parallel blinded design. In parallel double-blinding, both research teams and patients are unaware of the intervention type (Fain, 2013). We found confidence in the study integrity because the design and training of research assistants facilitated redirection of any questions to the Principal Investigator (PI). The Research Assistant (RA), when administering the intervention, was asked about the intervention content message. She/he had to explain that she did not know the message content, since the study was a double-blinded study.

Following initial enrollment, the researcher selfscrutinizes administration of protocol to ensure that the integrity and consistent quality of critical thinking related to recruitment, screening and enrollment is maintained. Those participants who enroll may be excluded if the researcher later finds out that protocol related to the enrollment is not followed. One example, in this study was that a participant was enrolled, and then on medical record review was found to have a diagnosis of dementia. Dementia or cognitive impairment was an exclusion criterion. In instances where study protocol was in question, protocols were reviewed and booster training of the research team was recommended, as was researcher assessment of the fidelity of the data collected.

\section{Conclusion}

Hospital staff, specifically unit staff, can identify potential older adult subjects based upon screening criteria. However, research in acute care hospitals' success is only possible if clinicians are familiar with how to facilitate the enrollment of patients and follow protocols. RCTs provide a necessary high standard for study integrity. Our RCT helped us identify the specific distinctions of older adults with respiratory diseases during the transition from acute care to community settings. Research is needed in areas that promote older adults' interest in enrolling in research studies, particularly RCT studies.

\section{Implications}

Health care providers and nurses must collaborate with hospitals and healthcare institutions, integrating the protocol into the internal workflow when conducting studies in hospital environments. Magnet accreditation, a standard for nursing care and research performance, has grown with currently 389 magnet hospitals in the United States, indicating increased nursing research activity aimed to identify optimal practices (American Nurses Credentialing Center, 2014). Therefore, magnet accreditation is a golden opportunity to engage health care providers, such as nurses, in evidence-based research among older adults in acute care hospitals (ACHs).

Our experiences suggest that securing access to an ACH to implement a RCT is a process of breaking down barriers to obtain permissions. An RCT requires collaboration and feedback from stakeholders and leaders at the hospitals. Older adult research in an $\mathrm{ACH}$ must ensure that 
care management is seamless and ethical, with protective measures for this vulnerable population. Understanding barriers to research promotes orchestration of a study. Increasing health care providers and nursing research actively involving older adults allows better insight into elderly decision-making capacity, comorbidities, and special needs, which can lead to better treatments, nursing care, and optimal health care.

\section{References}

American Nurses Credentialing Center. (2014). Growth of the program. Accessed July 27, 2014, from: http://www.nursecredentialing.org/Magnet/ProgramOverview/HistoryoftheMagnetProgram/ GrowthoftheProgram \& http://www.nursecredentialing.org/Magnet/ProgramOverview/HistoryoftheMagnetProgram.aspx

Administration on Aging. (2013). Profile of Older Americans. In Aging Statistics. Accessed July 27, 2014, from: http://www.aoa.gov/AoARoot/Aging_Statistics/Profile/index.aspx

Aujesky, D., \& Fine, M. J. (2008). The pneumonia severity index: A decade after the initial derivation and validation. Clinical Infectious Diseases, 47, S133-S139.

Barnett, K., Mercer, S. W., Norbury, M., Watt, G, Wyke, S., \& Guthrie B. (2012). Epidemiology of multimorbidity and implications for health care, research and medical education: A crosssectional study. Lancet, 380(9836), 38-43.

Cacioppo, J. T., \& Cacioppo, S. (2014). Older adults reporting social isolation or loneliness show poorer cognitive function 4 years later. Evidence-Based Nursing, 17(2), 59-60.

Clegg, A., Young, J., Iliffe, S., Rickkert, M. O., \& Rockwood, K. (2013). Frailty in elderly people. The Lancet, 381(9668), 752-762.

Dy, S. M. (2007). Instruments for evaluating shared medical decision making. Medical Care Research and Review, 64(6), 623-649.

Fain, J. A. (2013). Reading, understanding, and applying nursing research (4th ed., pp. 214-223). Phil, PA: F.A Davis Company.

Folstein, M. F., Folstein, S. E., \& McHugh, P. R. (1975). "Mini-mental state". A practical method for grading the cognitive state of patients for the clinician. Journal of Psychiatric Research, 12(3), 189-198.

Formiga, F., Ferrer, A., Sanz, H., Marengoni, A. l., Alburquerque, J., \& Pujol, R. (2012). Patterns of comorbidity and multimorbidity in the oldest old: The Octabaix study. European Journal of Internal Medicine, 24(1), 40-44.

Ganzini, L., Volicer, L., Nelson, W. A., Fox, E., \& Derse, A. R. (2005). Ten myths about decisionmaking capacity. Journal of the American Medical Directors Association, 6(Suppl_3), S100S104.

Hewner, S. (2014). A population-based care transition model for chronically ill elders. Nursing Economics, 32(3), 109-116.

Lessinger, M., Kulkarni, R., Zemans, R. L., Downey, G. P., \& Jeyasesian, S. (2014). Investigating the role of nucleotide-binding oligomerization domain like receptors in bacterial lung infection. Respiratory and Critical Care Medicine, 189(12), 1461-1468.

McHenry, J. C., Insel, K. C., Einstein, G. O., Vidrine, A. M., Koerner, K. M., \& Morrow, D. G. (2012). Recruitment of older adults: Success may be in the details. The Gerontologist, doi:10.1093/geront/gns079

Naylor, M. D., Brooten, D., Campbell, R., Jacobson, B. S., Mezey, M. D., Pauly, M. V., \& Schwartz, S. J. (1999). Comprehensive discharge planning and home follow-up of hospitalized elders: A randomized clinical trial. Journal of American Medical Association, 281(17), 613-620.

Naylor, M. D., Bowles, K. H., McCauley, K. M., Maccoy, M. C., Maislin, G., Pauly, M. V., \& Krakauer, R. (2011). High value transitional care: Transition of research into practice. Journal of Evaluation in Clinical Practice, 19(5), 1-7. 
Orwig, D., Rickles, N. M., \& Martin, L. G. (2011). Methodological issues in pharmacotherapy research in elderly adults. The American Journal of Geriatric Pharmacotherapy, 9(3), 173189.

Personality (2014). In Psychology Topics. Retrieved from: http://www.apa.org/topics/personality/

Polit, D. F., \& Beck, C. T. (2011). Nursing research: Generating and assessing evidence for nursing practice (8th ed., pp. 539-541). Philadelphia, PA: Lippincott Williams \& Wilkins.

Shaffer, S. T., Zarnowsky, C. D., Green, R. C., Lim, M. C., Holtzer, B. M., \& Ely, E. A. (2013). Strategies from bedside nurse perspectives in conducting evidence-based practice projects to improve care. Nursing Clinics of North America, 48(2), 353-361.

Tate, J. A., Snitz, B. E., Alvarez, K. A., Nahin, R. L., Weissfeld, L. A., Lopez, O., . . Y Yende, S.; GEM Study Investigators. (2014). Infection hospitalization increases risk of dementia in the elderly. Critical Care Medicine, 42(5), 1037-1046. doi:10.1097/CCM.0000000000000123

Verma, A. A., Razak, F., \& Detsky, A. S. (2014) Understanding choice: Why physicians should learn prospect theory. Journal of the American Medical Association, 311(6), 571-572.

Waltz, C., Strickland, O.L. \& Lenz, E. (2010) Measurement in Nursing Research. (4 ${ }^{\text {th }}$ ed.) New York, NY: Springer Publishing Co.

Wendler, M. C., Samuelson, S., Taft, L., \& Eldridge, K. (2011). Reflecting on research: Sharpening nurses' focus through engaged learning. Journal of Continuing Education in Nursing, 42(11), 487-493.

Author Information

Angela P. Halpin, PhDc, MSN, CNS, RN.

School of Nursing, University of California, Los

Angeles.

Contact: angela.halpin@ucla.edu

Felicia S. Hodge, DrPH. Committee Chairperson

School of Nursing and Public Health, University of

California, Los Angeles.

Contact: fhodge@sonnet.ucla.edu

Puiyen Chui, BSN student, research assistant.

School of Nursing, University of California, Los

Angeles

Contact: puiyenchui@ucla.edu 